'Departamento de Radiología, Clínica Las Condes, Santiago, Chile.

2Departamento de Cirugía, Clínica Las Condes, Santiago, Chile.

${ }^{3}$ Departamento de Anatomía Patológica, Clínica Las Condes, Santiago, Chile.

Recibido el 22 de junio de 2014, aceptado el 4 de diciembre de

2014

Correspondencia a: David Ladrón de Guevara Hernández

Clínica Las Condes, Servicio de Radiología $1^{\circ}$ piso. Lo Fontecilla 441, Las Condes. Teléfono: 22105174 Fax: 22105180 dlg@clc.c

\section{Rendimiento del Positron Emission Tomography/Computed Tomography (PET/CT) en etapificación de cáncer pulmonar}

\author{
DAVID LADRÓN DE GUEVARA H. ${ }^{1}$, FRANCISCA FURNARO L. ${ }^{1}$, \\ SEBASTIÁN YÉVENES A. ${ }^{1}$, JOSÉ MIGUEL CLAVERO R. ${ }^{2}$, \\ DAVID LAZO P. ${ }^{2}$, PATRICIO RODRÍGUEZ D. ${ }^{2}$, \\ ANTONIO PIOTTANTE B. ${ }^{3}$, RAÚl PEFAUR D. ${ }^{1}$, CLAUDIO PARDO B. ${ }^{1}$
}

\section{Positron emission tomography/computed tomography for lung cancer staging}

Background: PET/CT (Positron Emission Tomography/Computed Tomography) is widely used in nodal and metastatic staging of lung cancer patients. Aim: To analyze PET/CT detection of metastatic disease in patients with lung cancer. Material and Methods: We reviewed retrospectively ${ }^{F 18}$ Fluorodeoxyglucose PET/CT scans performed between December 2008 and December 2013. We selected 143 patients aged 30 to 92 years (63\% males) with confirmed lung cancer referred for staging, with no previous treatment. We reviewed whole body PET/CT and brain magnetic resonance images. SUVmax (Standardized Uptake Value maximum) of primary pulmonary lesion, hilar/mediastinal nodes, and distant metastases were calculated. Results: Histological types encountered were adenocarcinoma in 55\%, squamous-cell in 15\%, small-cell in 8\%, largecell in $6 \%$ and adeno-squamous in 2\%. In 22 cases (15\%) histology was not available. Nodal involvement was observed in $60 \%$ of patients (44\% hilar and 48\% mediastinal). Skip metastases (mediastinal involvement without hilum involvement) were encountered in $17 \%$ of cases, and were significantly more common among high uptake lung tumors $(p<0.01)$. Best SUVmax cut-off for node involvement was 4.4 for hilum and 4.0 for mediastinum (sensibility: $86.4 \%$, specificity: $99.8 \%)$. Sixty six patients (46.2\%) showed distant metastases on PET/CT. The most common metastases were osseous in $22 \%$, adrenal in $16 \%$, hepatic in $14 \%$, pulmonary in 14\% and cerebral in 12\%. PET/CT detected a second unexpected synchronic cancer in eight patients (6\%). Conclusions: PET/ CT is accurate for nodal staging using an uptake index as SUVmax. Distant metastases are common, especially in bone, adrenal glands and liver.

(Rev Med Chile 2015; 143: 22-29)

Key words: Lung neoplasms; Mediastinum; Neoplasms metastasis.
$\mathrm{E}$ 1 cáncer pulmonar es uno de las enfermedades malignas más frecuentes en el mundo, ocurriendo 1,8 millones de casos nuevos el año 2012, y constituye la primera causa de muerte por cáncer en el mundo, siendo responsable de 1,59 millones de fallecimientos al año ${ }^{1}$. A pesar de los avances en el tratamiento de estos pacientes, la sobrevida a 5 años sigue siendo pobre, aproximadamente de $16 \%{ }^{2,3}$. La existencia de metástasis al momento del diagnóstico, observada en $40-50 \%$ de los pacientes, es un factor determinante en el tratamiento y pronóstico de estos pacientes ${ }^{3}$. El PET/CT (Positron Emission Tomography/Computed Tomography) ha demostrado utilidad en el 
estudio inicial de estos pacientes y, en recientes publicaciones de costo-eficiencia, ha sido mencionado como una opción recomendable desde el punto de vista económico ${ }^{4,5}$. El aporte del PET/CT se debe principalmente a su elevado rendimiento en la etapificación mediastinica y en la detección de metástasis a distancia ${ }^{6}$. En etapificación ganglionar, el PET/CT presenta una sensibilidad de $79 \%$ vs $60 \%$ de la tomografía computada (TC) y especificidad de $91 \%$ vs $77 \%$ de la $\mathrm{TC}^{7}$. Por otro lado, el PET/CT puede detectar metástasis insospechadas en hasta $30 \%$ de los casos, y produce un cambio en el manejo clínico hasta en 55\% de los pacientes ${ }^{4,7}$. Por este motivo, el PET/CT es considerado actualmente el examen de imagen de elección en el estudio inicial de pacientes con cáncer pulmonar ${ }^{3}$.

El objetivo de este estudio es describir las características de la enfermedad metastásica ganglionar y a distancia en pacientes con cáncer pulmonar que se estudiaron con PET/CT en nuestro centro.

\section{Pacientes y Método}

\section{Pacientes}

Se revisaron retrospectivamente los PET/CT realizados en el Departamento de Radiología de Clínica Las Condes, entre diciembre de 2008 y diciembre de 2013, seleccionando aquellos efectuados a pacientes con cáncer pulmonar recientemente diagnosticado, sin tratamiento previo, y que acudían para etapificación. Antes de realizar el examen, se pesó, midió y entrevistó a cada paciente, constatando antecedentes clínicos relevantes.

\section{Adquisición del PET/CT}

Se realizó PET/CT previa inyección endovenosa $(0,1 \mathrm{mCi} / \mathrm{Kg})$ de $\mathrm{F} 18$-Fluorodeoxiglucosa (F18-FDG), utilizando equipo GE Discovery STE. En todos los pacientes se efectuó una tomografía computada de tórax, abdomen y pelvis con contraste iodado posterior al estudio PET, incluyendo el cerebro en los casos que no se habían realizado resonancia magnética $(\mathrm{RM})$ cerebral.

\section{Caracterización de las lesiones}

Se registró tamaño y ubicación de los tumores primarios pulmonares. La captación de F18-FDG del tumor primario pulmonar, ganglios hiliares, mediastínicos y extratorácicos, y metástasis a distancia fue cuantificada por medio del índice
SUVmax, de acuerdo al método descrito previamente $^{6,8}$. En mediastino, se estableció el mejor valor de corte de SUVmax para identificar ganglios positivos, basado en la confirmación histológica.

Las metástasis óseas fueron clasificadas por dos radiólogos expertos analizando tanto la TC como las imágenes del PET, en cuatro grupos según su aspecto: 1) líticas, en caso de destrucción ósea suficiente para ser detectada en la TC; 2) escleróticas, cuando existió esclerosis suficiente para ser vista en la TC; 3) mixtas, en caso de lisis y esclerosis asociadas en la misma lesión y 4) medulares, en lesiones con matriz ósea intacta, sólo visibles al PET.

Las lesiones no típicas de metástasis visualizadas con PET/CT y CTc fueron denominados hallazgos incidentales, y se clasificaron como: 1) verdadero positivo, cuando fue confirmado como otro tumor diferente al cáncer pulmonar; 2) falso positivo, cuando se confirmó como de naturaleza benigna y 3 ) no confirmado.

\section{Estudio histológico}

Se revisó la anatomía patológica de todos los pacientes, tanto referente al tumor primario, como al estudio ganglionar hiliar/mediastínico y lesiones extratorácicas. En caso de metástasis que no fueron biopsiadas, generalmente en pacientes con enfermedad metastásica avanzada, la confirmación de la naturaleza secundaria de dichas lesiones se realizó al comprobar la respuesta al tratamiento sistémico en PET/CT de control post terapia.

\section{Análisis estadístico}

Se utilizó curvas ROC, t-test y comparación de proporciones.

\section{Resultados}

Se seleccionaron 143 pacientes con cáncer pulmonar (mediana edad: 67 años, rango 30-92 años, $62,9 \%$ varones) comprobado histológicamente, sin tratamiento previo al PET/CT (Tabla 1).

De los 143 pacientes con cáncer pulmonar, $82,7 \%$ refirieron ser tabáquicos, y $17,3 \%$ nunca haber fumado. En ambos grupos, el tipo histológico adenocarcinoma fue el más frecuente $(85 \%$ de los no fumadores y $57 \%$ de los fumadores).

\section{Histopatología}

El 54,5\% (78/143) de los pacientes estudiados correspondieron a adenocarcinoma; $14,7 \%$ (21/143) a escamoso; 7,7\% (11/143) a células 
Tabla 1. Características de los pacientes y del tumor primario

\begin{tabular}{|c|c|}
\hline \multicolumn{2}{|l|}{ Edad } \\
\hline Mediana (rango) & 67 años (30-92 años) \\
\hline \multicolumn{2}{|l|}{ Género (n, \%) } \\
\hline Hombre & $90(62,9)$ \\
\hline Mujer & $53(37,1)$ \\
\hline \multicolumn{2}{|c|}{ Ubicación tumor primario (n, \%) } \\
\hline LSI & $44(30,8)$ \\
\hline LSD & $41(28,7)$ \\
\hline LM & $7 \quad(4,9)$ \\
\hline LII & $16(11,2)$ \\
\hline LID & $33(23,0)$ \\
\hline Mediastino & $2(1,4)$ \\
\hline Células Pequeñas (n, \%) & $11(7,7)$ \\
\hline Enf. Limitada & $5(45,5)$ \\
\hline Enf. Diseminada & $6 \quad(54,5)$ \\
\hline No células pequeñas (n, \%) & $132(92,3)$ \\
\hline I A & $33(25,0)$ \\
\hline I B & $4(3,0)$ \\
\hline$\| A$ & $8(6,1)$ \\
\hline II B & $4(3,0)$ \\
\hline III A & $15(11,4)$ \\
\hline III B & $5(3,8)$ \\
\hline IV & $63(47,7)$ \\
\hline
\end{tabular}

LSI: Lóbulo superior izquierdo; LSD: Lóbulo superior derecho; LM: Lóbulo medio; LII: Lóbulo inferior izquierdo; LID: Lóbulo inferior derecho. pequeñas; $5,6 \%(8 / 143)$ a células grandes y $2,1 \%$ (3/143) a adenoescamoso. En 22 casos, la histopatología no pudo ser recuperada, los que correspondieron principalmente a pacientes derivados de otros centros, sin ficha clínica en Clínica Las Condes. El índice SUVmax y tamaño del tumor primario pulmonar, y las metástasis ganglionar y a distancia, según grupo histológico se muestra en la Tabla 2.

\section{Etapificación mediastínica}

Se biopsiaron un total de 513 ganglios torácicos en 45 pacientes, correspondiendo a 33 lobectomías/segmentectomías y disección ganglionar, 10 biopsias transbronquiales con aguja fina guiadas por EBUS (Endobronchial ultrasound) y 2 mediastinoscopias. Al comparar con la histología, el mejor valor de corte de SUVmax para determinar positividad ganglionar fue de 4,4 para los linfonodos hiliares y 4,0 para los linfonodos mediastínicos, con una sensibilidad de $86,4 \%$, especificidad de $99,8 \%$, valor predictivo positivo de $97,4 \%$, valor predictivo negativo de $98,7 \%$, y rendimiento global de $98,6 \%$ (Tabla 3 ).

El 17,4\% presentó skip metastasis (compromiso mediastínico con hilio negativo). Dichos pacientes tuvieron tumores pulmonares con SUVmax significativamente más elevado (SUVmax: 19,7, DS. 17,1) que aquellos N2 sin skip metastasis (SUVmax: 13,9, DS: 6,7) (p: 0,03), sin diferencias significativas en el tamaño del tumor primario entre los pacientes con y sin skip metastasis.

Tabla 2. Captación de F18-FDG y tamaño del tumor primario, compromiso ganglionar y metástasis a distancia según tipo histológico del cáncer pulmonar

\begin{tabular}{|c|c|c|c|c|c|c|c|c|c|c|}
\hline & \multirow[t]{2}{*}{ Total } & \multicolumn{3}{|c|}{ SUVmax } & \multicolumn{2}{|c|}{ Diámetro } & \multicolumn{2}{|c|}{ Compromiso GGL } & \multicolumn{2}{|c|}{ MTT a distancia } \\
\hline & & Prom (DS) & rango & $\mathbf{p}^{*}$ & Prom (DS) & Rango & $\mathbf{n}$ & $\%$ & $\mathbf{n}$ & $\%$ \\
\hline$A D$ & 78 & $9,1 \quad(6,4)$ & $0,8-27,2$ & & $4,1(2,9)$ & $0,7-14,5$ & 37 & 47,4 & 37 & 47,4 \\
\hline AD-ESC & 3 & $18,8 \quad(7,0)$ & $11,3-25,0$ & p: 0,0121 & $5,5(2,6)$ & $2,9-8,0$ & 2 & 66,7 & 1 & 33,3 \\
\hline ESC & 21 & $18,8 \quad(7,8)$ & $5,3-40,2$ & $p<0,0001$ & $5,4(2,3)$ & $1,5-8,2$ & 15 & 71,4 & 7 & 33,3 \\
\hline GR & 8 & $19,6 \quad(9,6)$ & $4,4-31,0$ & $p<0,0001$ & $6,1(3,9)$ & $2,0-13,3$ & 7 & 87,5 & 5 & 62,5 \\
\hline PEQ & 11 & $15,3(19,1)$ & $3,5-71,9$ & p: 0,0322 & $4,6(3,0)$ & $1,1-8,6$ & 10 & 90,9 & 6 & 54,5 \\
\hline$S / H$ & 22 & $12,1 \quad(7,7)$ & $2,0-29,8$ & p: 0,0666 & $4,6(2,4)$ & $1,1-9,5$ & 15 & 68,2 & 10 & 45,5 \\
\hline Total & 143 & $12,3 \quad(9,3)$ & $0,8-71,9$ & & $4,6(2,8)$ & $0,7-14,5$ & 86 & 60,1 & 66 & 46,2 \\
\hline
\end{tabular}

AD: Adenocarcinoma; AD-ESC: Carcinoma adeno-escamoso; ESC: Carcinoma escamoso; GR: Carcinoma de células grandes; PEQ: Carcinoma de células pequeñas; S/H: Histología no recuperada; GGL: Ganglionar; MTT: Metástasis. *: Comparación con adenocarcinoma (t-test). 
PET/CT en etapificación de cáncer pulmonar - D. Ladrón de Guevara et al

Tabla 3. Rendimiento del PET/CT en etapificación N1-2 (valor de corte de SUVmax $\geq 4,4$ en hilio y $\geq 4,0$ en mediastino)

\begin{tabular}{|c|c|c|c|}
\hline & & \multicolumn{2}{|c|}{ PET } \\
\hline \multirow{4}{*}{ BP } & & GGL + & GGL - \\
\hline & + & 38 & 6 \\
\hline & - & 1 & 468 \\
\hline & & 39 & 474 \\
\hline \multicolumn{4}{|c|}{$p<0,0001$} \\
\hline
\end{tabular}

BP: Biopsia; GGL: Ganglio.

El mejor valor de corte para predecir skip metastasis fue de SUVmax $\geq 28$ en el tumor pulmonar primario. Incluyendo sólo los pacientes con mediastino positivo, la probabilidad de presentar skip metastasis fue de $15 \%$ para tumores con SUVmax menor a 28 , y de $67 \%$ para tumores con SUVmax $\geq 28$.

Considerando los pacientes con y sin metástasis a distancia, $60,1 \%$ de los individuos presentó compromiso ganglionar hiliar $(44,1 \%)$ y/o mediastinico (48,3\%). El 28,7\% (41/143) presentó compromiso ganglionar hiliar y/o mediastínico exclusivo, sin diseminación a distancia al PET/CT.

\section{Metástasis a distancia}

El 46,2\% (66/143) de los pacientes presentó metástasis a distancia al momento del diagnóstico. Las localizaciones más frecuentes se detallan en la Tabla 4. El adenocarcinoma mostró significativa (p: 0,02) mayor frecuencia de metástasis pulmonares $(19,2 \%)$ que el grupo no adenocarcinoma $(2,3 \%)$, sin otras diferencias en la distribución de la enfermedad metastásica entre los tipos histológicos.

Se contabilizaron un total de 218 metástasis óseas, $54,6 \%$ de las cuales fueron medulares, $23,9 \%$ líticas, $16,1 \%$ escleróticas y $5,4 \%$ mixtas. Es decir, más de la mitad de las metástasis óseas no provocaron alteración de la matriz ósea (medulares) y fueron visibles sólo con PET (Figura 1). Las lesiones líticas presentaron significativo (p: 0,001) mayor índice SUVmax (promedio: 14,1, DS: 7,0) que el grupo esclerótico-mixto-medular (SUVmax promedio: 7,4, DS: 3,4).

En 8 pacientes se observaron metástasis a distancia en ausencia de adenopatías hiliares o mediastínicas, 6 de ellos portadores de adeno-
Tabla 4. Captación de FDG y distribución de las metástasis en 143 pacientes con cáncer pulmonar

\begin{tabular}{|lcc|}
\hline & $\begin{array}{c}\text { SUVmax } \\
\text { Prom (DS) }\end{array}$ & $\begin{array}{c}\text { Total } \\
\mathbf{( \% )}\end{array}$ \\
\hline Óseo & $10,0(6,0)$ & $32(22,4)$ \\
SSRR & $8,1(4,2)$ & $23(16,1)$ \\
Hígado & $7,1(4,7)$ & $20(14,0)$ \\
Pulmón & $4,8(4,0)$ & $20(14,0)$ \\
Pleura & $3,8(3,3)$ & $20(14,0)$ \\
Cerebro & $10,4(3,5)$ & $17(11,9)$ \\
Muscular & $6,5(3,3)$ & $7(4,9)$ \\
\hline Tiroides & $6,7(1,8)$ & $4(2,8)$ \\
\hline Peritoneo & $8,1(5,1)$ & $4(2,8)$ \\
Renal & $10,9(1,8)$ & $3(2,1)$ \\
Retroperitoneo & $8,1(6,2)$ & $3(2,1)$ \\
\hline Pericardio & $3,7(0,8)$ & $3(2,1)$ \\
\hline Bazo & $3,8(1,0)$ & $2(1,4)$ \\
\hline Miocardio & 6,4 & $1(0,7)$ \\
\hline Páncreas & 8,1 & $1(0,7)$ \\
Cel. Subcutáneo & 12,9 & $1(0,7)$ \\
\hline
\end{tabular}

SSRR: glándulas suprarenales.

carcinoma de entre 1,7 y $5,7 \mathrm{~cm}$ y con SUVmax promedio 10,0 en el tumor primario pulmonar (rango 3,1-20,0). Es decir, 12,1\% (8/66) de los pacientes con metástasis hematógenas no tenía compromiso ganglionar detectable.

Los pacientes con metástasis a distancia fueron portadores de tumores pulmonares de significativo $(\mathrm{p}<0,0001)$ mayor tamaño (diámetro promedio: $5,8 \mathrm{~cm}$, DS: 2,7$)$ que aquellos sin metástasis (diámetro promedio: $3,4 \mathrm{~cm}$, DS: 2,4 ). No se demostró diferencias significativas en el SUVmax del tumor primario entre los pacientes con (promedio: 14,9, DS: 9,9) y sin metástasis a distancia (promedio: 9,7, DS: 7,9).

\section{Hallazgos incidentales del PET/CT}

Del total de hallazgos incidentales al PET/CT y TCc, 35,7\% (10/28) fueron confirmados como un segundo tumor primario en 8 pacientes, $57,1 \%$ (16/28) no fueron confirmados, y 7\% (2/28) correspondieron a falsos positivos del PET.

En 5,6\% (8/143) de los pacientes el PET/CT 

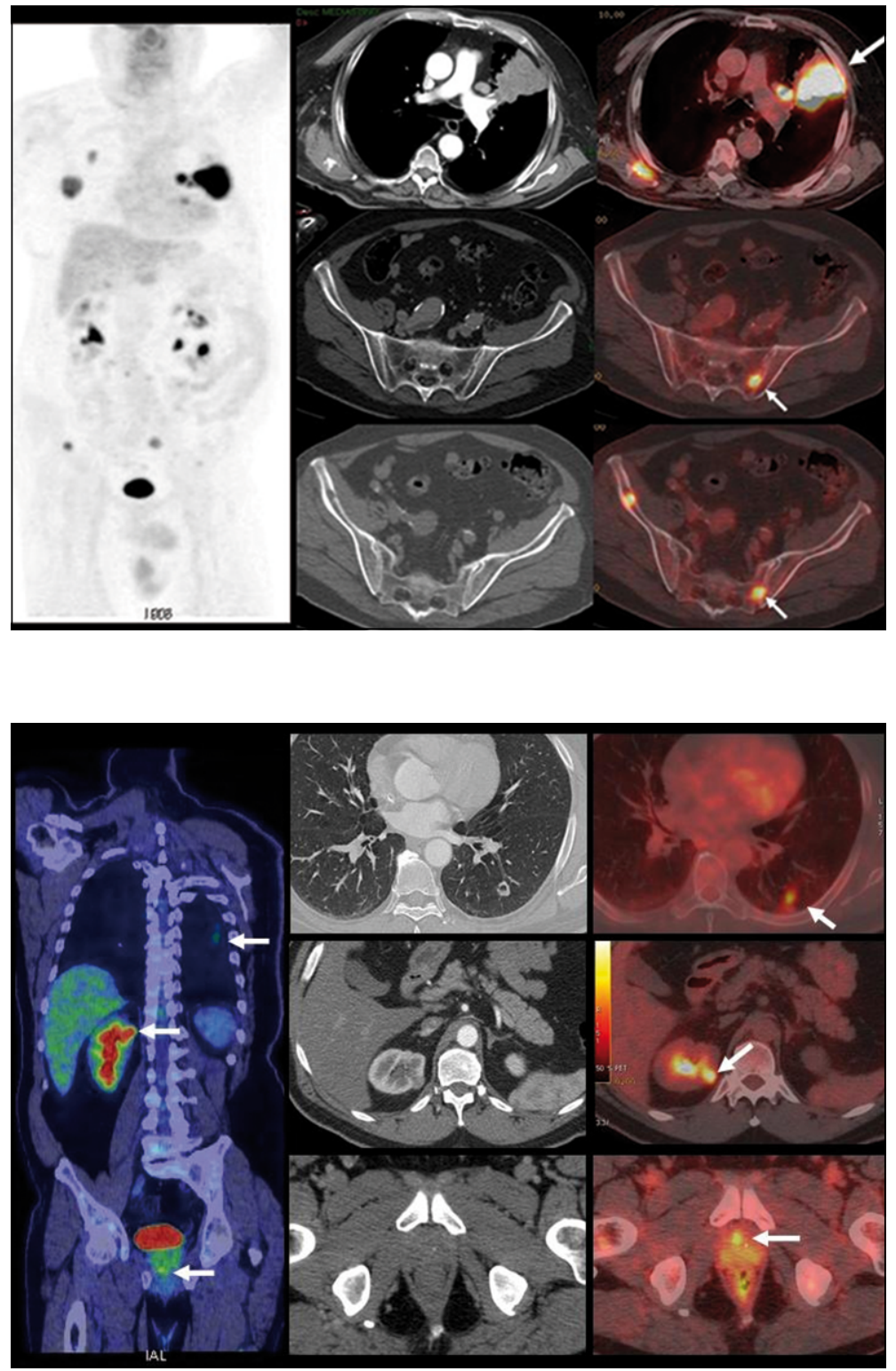

Figura 1. Adenocarcinoma pulmonar (flecha grande) con diseminación ganglionar y metástasis óseas medulares invisible a la tomografía computada (flechas pequeñas) y líticas que sí son evidentes a la TC.

Figura 2. Carcinoma escamoso pulmonar en el lóbulo inferior izquierdo (flecha en fotos superiores). El paciente presentó además nódulo renal derecho ávido de F18-FDG que resecó con resultados de carcinoma de células renales (flechas en fotos intermedias), y pequeño nódulo hipercaptante prostático que se operó con resultado de adenocarcinoma prostático (flechas en fotos inferiores).

detectó otras neoplasias malignas sincrónicas al cáncer de pulmón. Correspondieron a dos casos de cáncer de colon, ambos con lesiones parietales con intensa captación de FDG, dos casos de cáncer de células renales, uno de alta y otro de baja captación de FDG, dos casos con otros tumores pulmonares de diferentes características tomográficas y metabólicas que fueron confirmados con otra histología, dos casos con cáncer tiroideo, y uno con un cáncer de mama. Dos de estos pacientes fueron portadores de dos tumores malignos sincrónicos al cáncer de pulmón, es decir de tres neoplasias concomitantes (Figura 2).

\section{Discusión}

En pacientes con cáncer pulmonar, el grado de diseminación ganglionar hiliar y mediastinica, $y$ la existencia de metástasis a distancia determinan fuertemente la conducta respecto a los posibles 
tratamientos a considerar, particularmente la viabilidad de la opción quirúrgica. La existencia de enfermedad mediastinica contralateral (N3) o de siembra secundaria pulmonar, pleural o extratorácica alejan esta posibilidad. En series norteamericanas que incluyen tanto carcinoma de células pequeñas como de células no pequeñas, aproximadamente $22 \%$ de los pacientes presentan compromiso mediastinico exclusivo y $57 \%$ metástasis a distancia al momento del diagnóstico ${ }^{2,3}$. Esta cifra se acerca a lo obtenido en nuestra casuística, con $28 \%$ de compromiso ganglionar exclusivo y $46 \%$ de metástasis a distancia, lo que sugiere que el grupo estudiado sería representativo de la población con enfermedad diseminada.

El PET/CT ha mostrado un alto rendimiento en la etapificación ganglionar en neoplasias malignas, con una mayor sensibilidad que los métodos tradicionales, al poder detectar ganglios pequeños hipercaptantes de FDG que no califican como adenopatías en la TC o RM, y una mayor especificidad al diferenciar adenopatías reactivas o residuales, de alta frecuencia en hilio pulmonar y mediastino, de aquellas comprometidos por el tumor. La cuantificación de la captación de FDG en los linfonodos es esencial en su adecuada caracterización, y el índice SUV es el más empleado para este efecto. Valores de corte de entre 2,5 y 5,3 han sido mencionados como útiles para diferenciar ganglios reactivos/residuales de ganglios metastáticos, aunque estos valores pueden variar según el equipo PET/CT y la técnica de adquisición empleadas, por lo que se recomienda tener valores de corte propios en cada centro ${ }^{9,10}$. Los valores de SUVmax de 4,0 para mediastino y 4,4 para hilio obtenidos en este estudio apuntan en ese sentido, y permiten etapificar el mediastino con una sensibilidad de $86 \%$ y especificidad cercana a $100 \%$.

Aunque el compromiso mediastínico suele anteceder a la ocurrencia de metástasis a distancia, no es infrecuente encontrar siembra secundaria en ausencia de enfermedad ganglionar hiliar/ mediastinica ( $12 \%$ de los pacientes con metástasis en nuestra serie). El adenocarcinoma fue el tipo histológico mayormente involucrado en estos casos, tratándose a veces de tumores pequeños menores de $2 \mathrm{~cm}$ (T1a) que debutan con metástasis a distancia y que presentan mediastino negativo. Estos hallazgos apoyan la opción de etapificar en forma completa a pacientes aun en etapas $\mathrm{T}$ y $\mathrm{N}$ precoces de la enfermedad ${ }^{3}$.
Luego de la diseminación ganglionar, la segunda localización más frecuente de metástasis es el esqueleto, correspondiendo a $22 \%$ del total de pacientes en nuestra casuística. Dicho valor es coincidente con el $22 \%$ encontrado por Cheran et $\mathrm{al}^{11} \mathrm{y}$ dentro del rango $16-30 \%$ mencionado en la literatura ${ }^{12}$. El PET/CT ha mostrado significativa mayor rendimiento que la TC y que el cintigrama óseo en detección de metástasis óseas en cáncer de pulmón ${ }^{11,12}$. Esto se debe en parte al tipo de lesión ósea secundaria que predomina en el cáncer de pulmón, que puede no ser evidente con dichos exámenes de imagen. En nuestra serie, se observó una alta frecuencia de lesiones óseas medulares (54\%), que no son invisibles a la TC, ya que no modifican la matriz ósea, pero sin son visibles como hipercaptantes al PET. Por otro lado, un significativo número de lesiones fueron líticas (24\%), que son las que muestran los niveles más altos de captación de FDG, y que pudieran no ser vistas en estudios de cintigrama o PET óseo (PET con $\mathrm{NaF}$ ), ya que estos últimos basan su captación exclusivamente en la actividad osteoblástica (formación de hueso) y no osteoclástica ${ }^{13}$.

Las glándulas suprarrenales fueron la tercera localización más frecuente, presentes en $16 \%$ de los pacientes. En caso de lesiones suprarrenales dudosas es de utilidad medir la captación de FDG, ya que valores de SUVmax mayores a 2,5 pueden diferenciar las metástasis de los nódulos benignos $^{14}$.

Las metástasis hepáticas presentaron igual frecuencia que el pulmón y la pleura, y fueron siempre de alta captación de FDG. Esta característica es de suma importancia al querer diferenciar lesiones benignas hepáticas, de alta incidencia en la población adulta, de lesiones malignas secundarias y explica la alta especificidad del PET en lesiones focales del hígado ${ }^{4}$. Por otro lado, la técnica híbrida PET/CT potencia la sensibilidad de la TCc, que fluctúa entre 73 y $85 \%$, llevándola hasta $93-97 \%$ en la detección de metástasis hepáticas ${ }^{15,16}$.

Si bien las lesiones secundarias cerebrales no son las más frecuentes en esta población, su importancia radica en que su presencia determina una alta morbi-mortalidad, que en algunos casos puede ser evitada o al menos disminuida con un adecuado tratamiento. El PET/CT presenta una baja sensibilidad en la detección de metástasis cerebrales, determinada principalmente por la alta captación fisiológica cerebral, que hace más 
difícil distinguir las lesiones. Por este motivo, se recomienda complementar el estudio PET/CT con una RM cerebral en todos los pacientes con cáncer pulmonar desde la etapa $\mathrm{IB}^{3}$. En nuestra serie, todos los pacientes fueron estudiados con TC contrastada al momento de realizar el PET/ $\mathrm{CT}$, salvo que contaran con una RM cerebral previa, encontrándose metástasis encefálicas en $12 \%$ de los pacientes. Esta cifra es similar al 14\% encontrado en otras series estudiadas con $\mathrm{TCc}^{17}$.

La ocurrencia de una segunda neoplasia primaria sincrónica al cáncer pulmonar ha sido mencionada en algunas publicaciones, con frecuencias algo menores a la encontrada en nuestra muestra, entre 1,7 y $4,2 \%$ de los pacientes ${ }^{18-20}$. Ya que un número importante de los hallazgos incidentales del PET/CT no fueron confirmados histológicamente, es posible que nuestro estudio subestime la ocurrencia de segundas neoplasias primarias asociadas al cáncer pulmonar, e incluso de metástasis en ubicación inhabitual. Esto se constituye como una limitación de nuestro estudio, que se replica en otras publicaciones, donde $37-45 \%$ de los "incidentalomas" del PET/CT no son confirmados ${ }^{20}$.

\section{Conclusión}

El PET/CT presenta alto rendimiento en la etapificación ganglionar de pacientes con cáncer pulmonar utilizando índices de captación como el SUVmax. El mejor valor de corte para identificar los ganglios metastásicos fue un SUVmax $\geq$ 4,0 en mediastino $y \geq 4,4$ en hilio pulmonar. Las metástasis a distancia son de alta ocurrencia en esta población, siendo las más frecuentes las óseas, suprarrenales y hepáticas. El PET/CT muestra una elevada sensibilidad en la detección de lesiones secundarias en estas localizaciones, especialmente en el esqueleto, y permite detectar metástasis no visibles con la TCc. El 5,6\% de los pacientes con cáncer pulmonar presentó otro cáncer sincrónico al momento del diagnóstico, y el PET/CT es un excelente método en su detección.

Agradecimientos: Se agradece la encomiable colaboración del Dr. Álvaro Undurraga del Servicio Médico Quirúrgico Respiratorio del Instituto Nacional del Tórax en la recolección de datos clínicos de pacientes de esa institución.

\section{Referencias}

1. GLOBOCAN 2012. Estimated Cancer Incidence, Mortality and Prevalence Worldwide in 2012. http://globocan. iarc.fr.

2. Howlander N, Noone A, Krapcho M. SEER Cancer Statistics Review, 1975-2009 (Vintage 2009 Population) based on November 2001 SEER data submission. Bethesda, MD: National Cancer Institute 2012. http://seer.cancer. gov/statfacts/html/lungb.html

3. National Comprehensive Cancer Network guidelines 2014. http://www.nccn.org/professionals/physician_ gls/f_guidelines.asp\#site

4. Ambrosini V, Nicolini S, Caroli P, Nanni C, Massaro A, Marzola MC, et al. PET/CT imaging in different types of lung cancer: An overview. Eur J Radiol 2012; 81: 9881001.

5. American Cancer Society. Detailed guide: lung cancer non-small cell. What is non-small cell lung cancer? American Cancer Society 2010. http://www.cancer.org/ cancer/lungcancer-non-smallcell/detailedguide/

6. Ladrón de Guevara D, Pefaur R. PET/CT en cáncer pulmonar. Rev Med Chile 2010; 138: 1441-50.

7. Seltzer MA, Yap CS, Silverman DH, Meta J, Schiepers C, Phelps ME, et al. The impact of PET on the management of lung cancer: the referring physician's perspective. J Nucl Med 2002; 43: 752-6.

8. Ladrón de Guevara D, Fuentes A, Fariña C, Corral C, Pefaur R. Valor pronóstico del PET/CT en cáncer pulmonar. Estudio de sobrevida y caracterización metabólica tumoral. Rev Med Chile 2013; 141: 41-8.

9. Bryant AS, Cerfolio RJ, Klemm KM, Ojha B. Maximum Standard Uptake Value of Mediastinal Lymph Nodes on Integrated FDG PET-CT Predicts Pathology in Patients with Non-Small Cell Lung Cancer. Ann Thorac Surg 2006; 82: 417-23.

10. Enoch Lee B, Redwine J, Foster C, Abella E, Lown T, Lau $\mathrm{D}$, et al. Mediastinoscopy might not be necessary in patients with non-small cell lung cancer with mediastinal lymph nodes having a maximum standardized uptake value of less than 5,3. J Thorac Cardiovasc Surg 2008; 135: 615-9.

11. Cheran SK, Herndon JE, Patz EF. Comparison of wholebody FDG-PET to bone scan for detection of bone metastases in patients with a new diagnosis of lung cancer. Lung Cancer 2004; 44: 317-25.

12. Silvestri GA, Gould MK, Margolis ML, Tanoue LT, McCrory D, Toloza E, et al. Noninvasive Staging of Nonsmall Cell Lung Cancer: ACCP Evidenced-Based Clinical Practice Guidelines (2nd Edition). CHEST 2007; 132: 178S-201S. 
13. The Musculoskeletal System. In: Wagner HN, Szabo Z and Buchanan JW. Principles of Nuclear Medicine. Philadelphia, USA. W.B. Saunders Company, Second Edition 1995; 986-1020.

14. Brady MJ, Thomas J, Wong TZ, Franklin KM, Ho LM, Paulson EK. Adrenal Nodules at FDG PET/CT in Patients Known to Have or Suspected of Having Lung Cancer: A Proposal for an Efficient Diagnostic Algorithm. Radiology 2009; 250: 523-30.

15. Seo HJ, Kim MJ, Lee JD, Chung WS, Kim YE. Gadoxetate disodium-enhanced magnetic resonance imaging versus contrast-enhanced 18F-fluorodeoxyglucose positron emission tomography/computed tomography for the detection of colorectal liver metastases. Invest Radiol 2011; 46: 548-55.

16. Donati OF, Hany TF, Reiner CS, von Schulthess GK, Marincek B, Seifert B, et al. Value of retrospective fusion of PET and MR images in detection of hepatic metastases: comparison with 18F-FDG PET/ CT and Gd-EOBDTPA-enhanced MRI. J Nucl Med 2010; 51: 692-9.

17. Salbeck R, Grau HC, Artmann H. Cerebral Tumor Staging in Patients With Bronchial Carcinoma by Computed Tomography. Cancer 1990; 66: 2007-11.

18. Lardinois D, Weder W, Roudas M, von Schulthess GK, Tutic M, Moch H, et al. Etiology of Solitary Extrapulmonary Positron Emission Tomography and Computed Tomography Findings in Patients With Lung Cancer. J Clin Oncol 2005; 23: 6846-53.

19. De Wever W, Vankan Y, Stroobants S, Verschakelen J. Detection of extrapulmonary lesions with integrated $\mathrm{PET} / \mathrm{CT}$ in the staging of lung cancer. Eur Respir J 2007; 29: 995-1002.

20. Chopra A, Ford A, De Noronha R, Matthews S. Incidental findings on positron emission tomography/CT scans performed in the investigation of lung cancer. $\mathrm{Br}$ J Radiol 2012; 85: e229-37. 